\title{
Foam flow turn influence on the in-line tube bundle heat transfer intensity
}

\author{
J. Gylys ${ }^{1}$, S. Sinkunas ${ }^{2}$, T. Zdankus ${ }^{1}$, V. Giedraitis ${ }^{2} \&$ A. Balcius ${ }^{2}$ \\ ${ }^{1}$ Energy Technology Institute, Kaunas University of Technology, \\ Lithuania \\ ${ }^{2}$ Department of Thermal and Nuclear Energy, \\ Kaunas University of Technology, Lithuania
}

\begin{abstract}
An experimental investigation of heat transfer from the tubes to the two-phase foam system was performed. Statically stable gas-liquid foam flow was used as a coolant. An investigation was performed on the experimental laboratory set-up consisting of the foam generator, an experimental channel and tube bundles. Two different geometries of in-line tube bundles were used for the experiments. Regularities of heat transfer of the tube bundles to the foam flow under the $180^{\circ}$ degree turn were analysed in the work. The results of the investigation showed that heat transfer intensity is much higher than that for the one-phase airflow under the same conditions. The heat transfer character of frontal and further tubes to downward foam flow is different in comparison with the one-phase coolant flow. After the turn, local void fraction of the foam is less on the inner side of the foam flow. Therefore heat transfer intensity of the inner side-line tubes is higher than for other tubes of the bundle. The results of the investigation were generalized by criterion equations, which can be used for the calculation and design of the statically stable gas-liquid foam heat exchangers with the in-line tube bundles.
\end{abstract}

Keywords: statically stable foam, foam flow, flow turn, heat transfer, in-line tube bundle.

\section{Introduction}

One-phase coolants are usually used for heat and mass transfer processes in heat exchangers. Smaller coolant mass flow rate, relatively large heat transfer rate, low energy consumption required for coolant delivery to the heat transfer place 
may be achieved by usage of two-phase gas-liquid foam flow as a coolant. Nevertheless some problems appear in that case. First of all, heat transfer of different tube bundles to one-phase fluids was investigated [1, 2], but practically there aren't data related to the tube bundle heat transfer to the foam flow. Another problem is that a structure of the foam may not significantly vary while it passes different obstacles (turnings, tube bundles and so on), especially during the heat transfer process. Statically stable foam (one type of gas-liquid foam) keeps its initial structure and bubbles' dimension within broad limits of a long time interval even after termination of the foam generation and therefore is available for heat transfer purposes [3]. This type of foam can be generated from the solutions, which have less than pure liquid surface tension. However a number of foam peculiarities, drainage of liquid from foam [4, 5], diffusive transfer of gas between bubbles [6], division and collapse of foam bubbles [5], complicates an application of the analytical methods for heat transfer investigation. Therefore an experimental method of investigation was selected in our work as the most suitable.

Typical heat exchangers usually consist of several vertical parts in which coolant changes its direction from vertical upward to vertical downward and vice versa. Tube bundles of different types and geometry may be used in heat exchangers also. Therefore we performed an investigation of staggered $[7,8]$ and in-line [9] tube bundle heat transfer to vertical upward and downward after turning foam flow.

The main task of this work was to investigate experimentally and compare heat transfer intensity of two in-line tube bundles to vertical downward after $180^{\circ}$ degree turning foam flow. The results of our experimental investigation are presented and discussed in this paper.

\section{Experimental set-up}

Two in-line tube bundles were used during the experimental investigation. A schematic view of the experimental channel with tube bundles is shown in Fig. 1. The in-line tube bundle No. 1 consisted of five vertical rows with six tubes in each (Figure 1a). Spacing between centres of the tubes was $s_{1}=s_{2}=0.03 \mathrm{~m}$. The in-line tube bundle No. 2 consisted of five vertical rows with three tubes in each (Figure 1b). Spacing between centres of the tubes across the experimental channel was $s_{l}=0.03 \mathrm{~m}$ and spacing along the channel was $s_{2}=0.06 \mathrm{~m}$. External diameter of all the tubes was equal to $0.02 \mathrm{~m}$. An electrically heated tube calorimeter had an external diameter equal to $0.02 \mathrm{~m}$ also. During the experiments the calorimeter replaced one tube of the bundle. An electric current value of the heated tube was measured by an ammeter and the voltage by a voltmeter. The temperature of the calorimeter surface was measured by eight calibrated thermocouples: six of them were placed around the central part of the tube and two of them were placed in both sides of the tube at a distance of 50 $\mathrm{mm}$ from the central part. The temperature of the foam flow was measured by two calibrated thermocouples: one in front of the bundle and one behind it. 
The experimental set-up consisted of the following main parts: experimental channel, tube bundle, gas and liquid control valves, gas and liquid flow meters, liquid storage reservoir, liquid level control reservoir, air fan, electric current transformer and stabilizer [7-9]. The cross section of the experimental channel had dimensions $0.14 \times 0.14 \mathrm{~m}^{2}$; the height of it was $1.8 \mathrm{~m}$. The radius $(R)$ of the channel turning was equal to $0.17 \mathrm{~m}$.

Statically stable foam flow was used for an experimental investigation. This type of foam was generated from water solution of detergents. Concentration of detergents was kept constant and was equal to $0.5 \%$. Foam flow was produced during gas and liquid contact on the riddle, which was installed at the bottom of the experimental channel. Liquid was delivered from the reservoir to the riddle from the upper side; gas was supplied to the riddle from below.

Measurement accuracies for flows, temperatures and heat fluxes were in the ranges $1.5 \%, 0.15 \div 0.20 \%$ and $0.6 \div 6.0 \%$ correspondingly.

a)

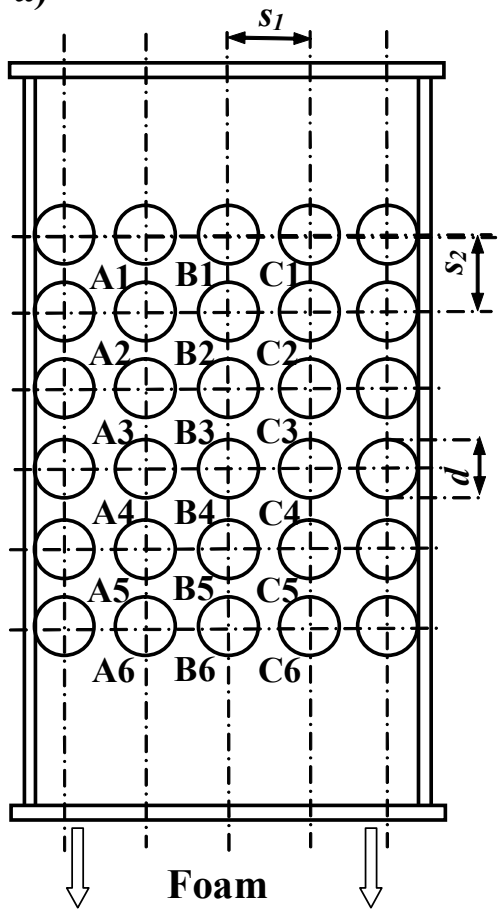

b)

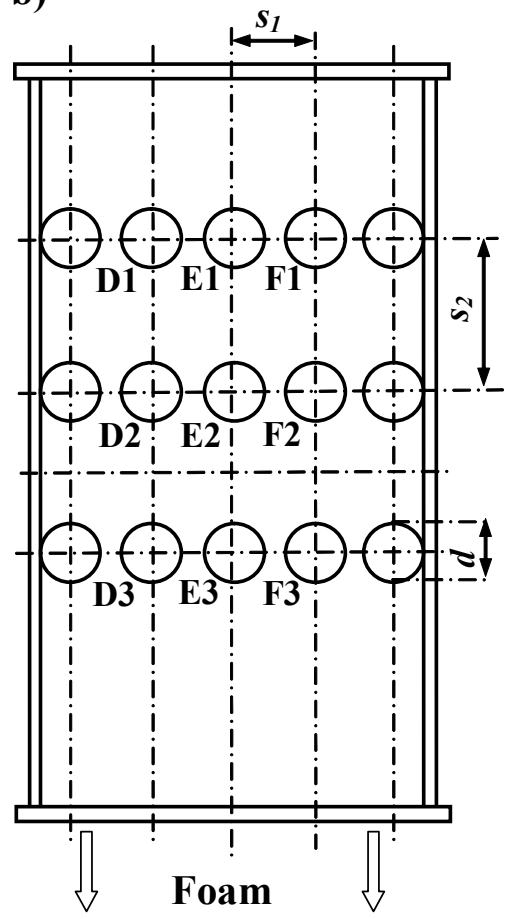

Figure 1: $\quad$ In-line tube bundle No. 1 (a) and No. 2 (b) in downward foam flow.

During the experimental investigation a relationship was obtained between an average heat transfer coefficient $h$ from one side and foam flow volumetric void fraction $\beta$ and gas flow Reynolds number $R_{g}$ from the other side:

$$
N u_{f}=f\left(\beta, \operatorname{Re}_{g}\right) .
$$


The Nusselt number was computed by the formula

$$
N u_{f}=\frac{h d}{\lambda_{f}}
$$

where $\lambda_{f}$ is the thermal conductivity of the statically stable foam flow, $\mathrm{W} /(\mathrm{m} \cdot \mathrm{K})$, computed by the equation

$$
\lambda_{f}=\beta \lambda_{g}+(1-\beta) \lambda_{l} .
$$

An average heat transfer coefficient was calculated as

$$
h=\frac{q_{w}}{\overline{\Delta T}} .
$$

The gas Reynolds number of foam flow was computed by the formula

$$
\operatorname{Re}_{g}=\frac{G{ }_{g} d}{A v_{g}} .
$$

Foam flow volumetric void fraction can be expressed by the equation

$$
\beta=\frac{G_{g}}{G_{g}+G_{l}} .
$$

Experiments were performed within Reynolds number diapason for gas $\left(R e_{g}\right)$ : $190 \div 440$ (laminar flow regime) and foam volumetric void fraction $(\beta)$ : $0.996 \div 0.998$. Gas velocity for foam flow was changed from 0.14 to $0.32 \mathrm{~m} / \mathrm{s}$.

\section{Results}

After statically stable foam generation on the riddle the foam flow was directed vertically upward then made a $180^{\circ}$ degree turn (radius of the turn was equal to $0.17 \mathrm{~m}$ ) and moved downward crossing the tube bundle. Initially an experiments with in-line tube bundle No. 1 was performed; then the tube bundle No. 2 was placed instead of the previous bundle and experiments followed.

Three main parameters of the foam flow influence the heat transfer intensity of different tubes of the bundles: foam structure, distribution of local flow velocity and distribution of local foam void fraction across and along the experimental channel. The liquid drainage process influence the distribution of the foam local void fraction and accordingly the heat transfer intensity of the tubes. Liquid drainage from foam phenomena depends on gravity and capillary. In a vertical direction these forces are acting together. In a horizontal direction the influence of gravity forces is negligible and the influence of capillary forces is dominating. Influence of the electrostatic and molecular forces on drainage is insignificant. Gravity forces act along the upward and downward foam flow. While foam flow makes a turn the gravity forces act across and along the foam flow. Liquid drains down from the upper channel wall and the local void fraction increases (foam becomes drier) here as well. After the turn, the local void fraction of foam is less (foam is wetter) on the inner-left side of the cross-section (tubes A and D, Fig. 1). 
Investigation with one-phase flow [1, 2] and our previous investigation with foam flow showed that heat transfer of the bundles' third and further tubes varies insignificantly [7-9]. Comparison of heat transfer intensity of the A3, B3, C3 and D3, E3, F3 tubes of the in-line bundles to downward after turning foam flow at the volumetric void fraction $\beta=0.997$ is shown in Fig. 2.

The heat transfer intensity of the third tubes of the in-line bundle No. 2 is higher than that of bundle No. 1. Increasing foam flow gas Reynolds number $\left(R e_{g}\right)$ from 190 to 440 , heat transfer intensity $\left(N u_{f}\right)$ of the tube A3 increases by 1.9 times (from 488 to 904 ), by 2.1 times (from 366 to 783 ) of the tube B3, and by 2.1 times (from 231 to 496 ) of the tube $\mathrm{C} 3$ for foam volumetric void fraction $\beta=0.997$. The heat transfer intensity of the tubes D3 for the same $R e_{g}$ increases by 1.8 times (from 637 to 1118 ), by 2.2 times (from 373 to 825 ) of the tube E3, and by 2.4 (from 252 to 617 ) of the tube F3 for $\beta=0.997$. When $R e_{g}=440$ the heat transfer intensity of the A3 tube is higher than that of the tube C3 and the heat transfer intensity of the D3 tube is higher than that of the tube F3 by 1.8 times.

The heat transfer intensity of the tube D3 is higher than that of the tube A3 on average by $26 \%$, the heat transfer of the tube E3 is higher than that of the tube $\mathrm{B} 3$ on average by $4 \%$, and the heat transfer of the tube F3 is higher than that of the tube C 3 on average by $20 \%$ for $\beta=0.997$ and $R e_{g}=190 \div 440$.

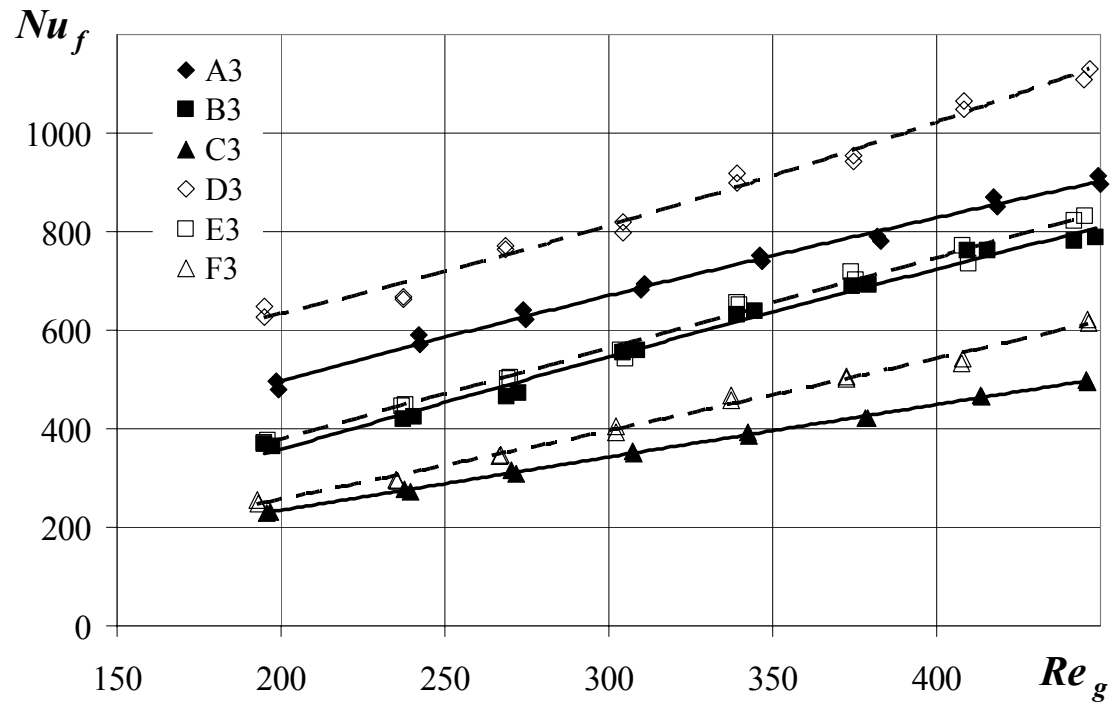

Figure 2: $\quad$ Heat transfer of the tubes A3, B3, C3 and D3, E3, F3 in downward foam flow, $\beta=0.997$. 


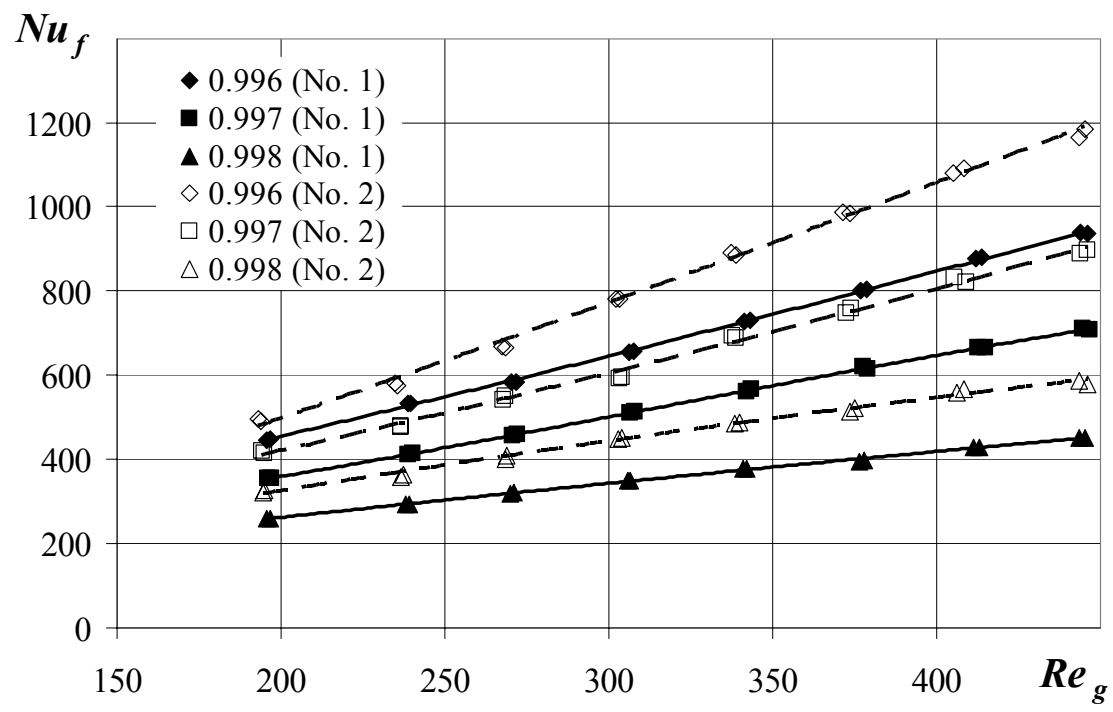

Figure 3: Average heat transfer of the tubes of the in line bundle No. 1 and No. 2 to downward foam flow: $\beta=0.996,0.997$ and 0.998 .

An average heat transfer rate was calculated in order to analyse and compare the experimental results of different in-line tube bundles. The average heat transfer intensity of the tubes of the in-line bundle No. 1 and No. 2 to downward after turning foam flow is shown in Fig. 3.

The effect of a "shadow" takes place in the case of the in-line bundle No. 1 and the average heat transfer intensity of the tubes of the in-line bundle No. 2 is higher than that of the tubes of the in-line bundle No. 1 for the whole interval of $R e_{g}\left(R e_{g}=190 \div 440\right)$. Changing $R e_{g}$ from 190 to 440 , the average heat transfer intensity of the tubes of the in-line bundle No. 1 to downward foam flow increases by 2.1 times for $\beta=0.996$; twice for $\beta=0.997$, and by 1.7 times for $\beta=0.998$; and that for the tubes of the in-line bundle No. 2 is by 2.4 times for $\beta=0.996$; by 2.1 times for $\beta=0.997$, and by 1.8 times for $\beta=0.998$.

The average heat transfer intensity of the tubes of the in-line bundle No. 2 is higher than that of the tubes of the in-line bundle No. 1 on average by $21 \%$ for $\beta=0.996$, by $23 \%$ for $\beta=0.997$ and by $27 \%$ for $\beta=0.998$ to downward foam flow for the whole interval of $\operatorname{Re}_{g}\left(\operatorname{Re}_{g}=190 \div 440\right)$.

Experimental results of investigation of heat transfer of the in-line tube bundles to downward after $180^{\circ}$ turning statically stable foam flow were generalized by the criterion equation using the dependence between the Nusselt number $N u_{f}$ and the gas Reynolds $R e_{g}$ number. This dependence within the interval $190<\operatorname{Re}_{g}<440$ for the in-line tube bundle in downward foam flow with the volumetric void fraction $\beta=0.996,0.997$, and 0.998 can be expressed as follows: 


$$
N u_{f}=c \beta^{n} \operatorname{Re}_{g}^{m} .
$$

On average, for the whole in-line tube bundle No. $1\left(s_{I}=s_{2}=0.03 \mathrm{~m}\right)$ in the downward foam flow $c=12.7, n=334, m=114.6(1.004-\beta)$.

On average, for the whole in-line tube bundle No. $2\left(s_{1}=0.03\right.$ and $\left.s_{2}=0.06 \mathrm{~m}\right)$ in the downward foam flow $c=22.4, n=675, m=167.8(1.002-\beta)$.

\section{Conclusions}

Heat transfer of two in-line tube bundles with different geometry to vertical laminar downward statically stable foam flow was investigated experimentally.

The liquid drainage process significantly transforms the "cross-sectional" distribution of the local void fraction of the downward foam flow and acts on the heat transfer intensity of the tubes.

Therefore, the heat transfer intensity of the left (A and D) side-line tubes is higher than that of the middle ( $\mathrm{B}$ and $\mathrm{E})$ and right $(\mathrm{C}$ and $\mathrm{F})$ side-line tubes.

The effect of a "shadow" is slight and heat transfer is higher for the tubes of the in-line tube bundle with more spacing between the tube centres along the bundle.

Results of the investigation were generalized by criterion equations, which can be used for the calculation and design of the statically stable foam heat exchangers with in-line tube bundles.

\section{Nomenclature}

$A$ - cross section area of experimental channel, $\mathrm{m}^{2} ; c, m, n$ - coefficients; $d-$ outside diameter of tube, m; $G$ - volumetric flow rate, $\mathrm{m}^{3} / \mathrm{s} ; N u$ - Nusselt number; $q$ - heat flux density, $\mathrm{W} / \mathrm{m}^{2} ; R e-$ Reynolds number; $\bar{T}-$ average temperature, $\mathrm{K} ; h$ - average coefficient of heat transfer, $\mathrm{W} /\left(\mathrm{m}^{2} \cdot \mathrm{K}\right) ; \beta-$ volumetric void fraction; $\lambda$ - thermal conductivity, $\mathrm{W} /(\mathrm{m} \cdot \mathrm{K}) ; v-$ kinematic viscosity, $\mathrm{m}^{2} / \mathrm{s}$.

\section{Indexes}

$f$-foam;

$g$ - gas;

$l$ - liquid;

$w$ - wall of heated tube.

\section{References}

[1] Zukauskas A., Convectional Heat Transfer in Heat Exchangers, Nauka: Moscow, p. 472, 1982.

[2] Hewitt, G. F., Heat exchanger design handbook 2002, York, Begell House, 2002. 
[3] Gylys, J., Hydrodynamics and Heat Transfer Under the Cellular Foam Systems, Technologija: Kaunas, 1998.

[4] Fournel B., Lemonnier H., Pouvreau J., Foam Drainage Characterization by Using Impedance Methods, 3rd Int. Symp. on Two-Phase Flow Modelling and Experimentation, Pisa, Italy, p. [1-7], 2004.

[5] Sadoc, J. F., Rivier, N., Foams and Emulsions, Nato ASI Series, 1997.

[6] Tichomirov V., Foams. Theory and Practice of Foam Generation and Destruction, Chimija: Moscow, 1983.

[7] Gylys J., Miliauskas G., Sinkunas S., Zdankus T., Influence of vertical foam flow liquid drainage on tube bundle heat transfer intensity, The Fourth International Conference on Heat Transfer, Fluid Mechanics and Thermodynamics, Cairo, Egypt, p. [1-6], 2005.

[8] Gylys J., Sinkunas S. and Zdankus T., Experimental Study of Staggered Tube Bundle Heat Transfer in Foam Flow, 5th International Symposium on Multiphase Flow, Heat Mass Transfer and Energy Conversion, Xi'an, China, p.[1-6], 2005.

[9] Gylys J., Giedraitis V., Sinkunas S., Zdankus T. and Gylys M., Study of in-line tube bundle heat transfer in upward vertical foam flow, Energy: production, distribution and conservation ASME conference, Milan, Italy, pp. 643-650, 2006. 